\title{
A COLLAPSE RESULT FOR THE EILENBERG-MOORE SPECTRAL SEQUENCE
}

\author{
BY HANS J. MUNKHOLM
}

Communicated by Saunders Mac Lane, April 17, 1972

1. Introduction. In this note we outline a proof of the following result. Details will appear elsewhere.

THEOREM. Let $F \rightarrow E \rightarrow^{p} B$ be a fibration with $E$ and $B$ 1-connected. $H^{*}\left(E ; Z_{2}\right)$ and $H^{*}\left(B ; Z_{2}\right)$ polynomial algebras in finitely many variables. Suppose also that $S q^{n-1}(y)=0$ for all $y \in H^{n} E$. Then the Eilenberg-Moore spectral sequence with $E_{2}=\operatorname{Tor}_{H^{*}\left(B ; Z_{2}\right)}\left(H^{*}\left(E ; Z_{2}\right), Z_{2}\right)$ and $E_{r} \Rightarrow H^{*}\left(F ; Z_{2}\right)$ collapses.

REMARKS. 1. The above applies very often to homogeneous spaces (take $G / H \rightarrow B H \rightarrow B G$ ).

2. For related results see e.g. [1], [2], [3], [4], [5], [6]. The main difference between our results and these earlier ones is that we do not have to impose conditions on the spaces, only on their cohomology.

3. Some evident generalizations (other coefficients, infinitely many generators for the polynomial algebras, fiber squares) are currently being worked out.

2. Outline of proof. Consider the category $\mathfrak{S G H}_{\mathfrak{A}}$ of differential graded algebras and shm maps, as defined in [7] (we impose some rather obvious extra normalization conditions). Write $f: A \Rightarrow B$. The category $\mathfrak{A}$ of differential graded algebras and multiplicative maps is the full subcategory determined by the condition $f_{i}=0$ for $i>1$. Let $I^{*}$ be the normalized cochains on the semisimplicial 1-simplex. Define a homotopy (from $f$ to g) to be an shm map $H: A \Rightarrow B \otimes I^{*}$ such that

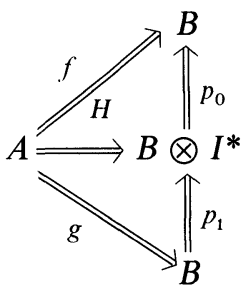

commutes. Here $p_{i}: B \otimes I^{*} \Rightarrow B$ are the obvious multiplicative projections. There is then the associated homotopy category $\mathfrak{S G H}_{h}$. Given 
$g: A \Rightarrow A^{1}, f: B \Rightarrow B^{1}$, one defines $A \otimes f: A \otimes B \Rightarrow A \otimes B^{1}$ by

$$
f_{i}\left(\left(a_{1} \otimes b_{1}\right) \otimes \cdots \otimes\left(a_{i} \otimes b_{i}\right)\right)=a_{1} a_{2} \cdots a_{i} \otimes f_{i}\left(b_{1} \otimes b_{2} \otimes \cdots \otimes b_{i}\right) .
$$

$g \otimes B^{1}: A \otimes B^{1} \Rightarrow A^{1} \otimes B^{1}$ is defined similarly and one puts $g \otimes f$ $=\left(g \otimes B^{1}\right)(A \otimes f)$. Then $\otimes$ is a functor in each variable separately on

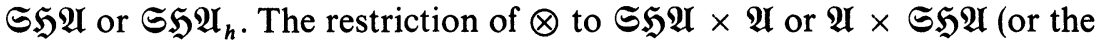
corresponding homotopy categories) is a bifunctor.

If $f: A \Rightarrow B$ then one may define Tor $(f)$ as in [7, Definition 12 and the remark following it]. If $f$ is multiplicative one has $\operatorname{Tor}(f)=\operatorname{Tor}_{A}\left(Z_{2}, B\right)$. There is the usual spectral sequence with $E_{2}=\operatorname{Tor}(H f)$ and $E_{r} \Rightarrow \operatorname{Tor}(f)$. As noted in [7] this Tor is functorial enough to get

$$
\operatorname{Tor}(f) \rightarrow \operatorname{Tor}(g f) \rightarrow \operatorname{Tor}(g)
$$

whenever one has $A \Rightarrow^{f} B \Rightarrow^{g} C$. On the $E_{2}$ level of the spectral sequences the corresponding maps are $\operatorname{Tor}_{H A}\left(Z_{2}, H g\right): \operatorname{Tor}_{H A}\left(Z_{2}, H B\right) \rightarrow$ $\operatorname{Tor}_{H A}\left(Z_{2}, H C\right)$ and $\operatorname{Tor}_{H f}\left(Z_{2}, H C\right): \operatorname{Tor}_{H A}\left(Z_{2}, H C\right) \rightarrow \operatorname{Tor}_{H B}\left(Z_{2}, H B\right)$, respectively, so if $H g$ (or $H f$ ) is an isomorphism then so is $\operatorname{Tor}(f) \rightarrow \operatorname{Tor}(g f)$ (or $\operatorname{Tor}(g f) \rightarrow \operatorname{Tor}(g)$ ).

It follows that when $H: f \simeq g: A \Rightarrow B$ one gets isomorphisms $\operatorname{Tor}(f)$ $\leftarrow \simeq \operatorname{Tor}(H) \rightarrow^{\simeq \operatorname{Tor}(g) \text {. Therefore, if }}$

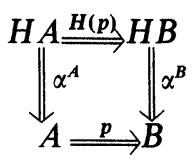

commutes in $\mathfrak{S} \mathfrak{H A}_{h}$ and $H\left(\alpha^{B}\right), H\left(\alpha^{A}\right)$ are isomorphisms then one gets a string of isomorphisms $\operatorname{Tor}(H(p)) \rightarrow{ }^{\simeq} \operatorname{Tor}\left(\alpha^{B} H(p)\right) \cong \operatorname{Tor}\left(p \alpha^{A}\right) \rightarrow \stackrel{\simeq}{\operatorname{Tor}}(p)$. and the corresponding spectral sequence collapses.

In [7] an shc algebra is defined to be a pair $(A, \Phi)$ where $\Phi: A \otimes A \Rightarrow A$ has $\Phi_{1}=\Phi_{A}$. It is noted that one has the iterates $\Phi^{n}: A^{(n)} \Rightarrow A$ and that $A^{(n)}$ again becomes an shc algebra in the obvious way. We shall further require that an shc algebra have

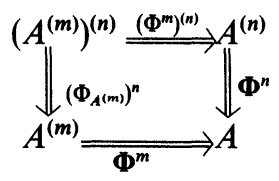

commutative in $\mathfrak{S H}_{\mathfrak{h}}$. Together with multiplicative maps $f: A \rightarrow B$ making

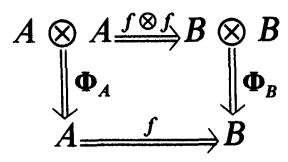


commutative in $\mathfrak{S} \mathfrak{S} \mathfrak{U}_{h}$ our shc algebras form the category shc $\mathfrak{A}$.

An acyclic model argument shows that cochains on simply connected semisimplicial sets form a functor into shc $\mathfrak{A}$.

If $H^{*} X=Z_{2}\left[z_{1}, \ldots, z_{k}\right]$ there is a multiplicative map $\beta^{X}: H^{*} X$ $\rightarrow\left(C^{*} X\right)^{(k)}$ sending $z_{i}$ to $1 \otimes \cdots \otimes \zeta_{i} \otimes \cdots \otimes 1$ where $\zeta_{i}$ represents $z_{i}$. The composition of $\beta^{X}$ with $\left(\Phi_{C^{*} X}\right)^{k}$ is then a homology isomorphism $\alpha^{X}: H^{*} X \Rightarrow C^{*} X$. Thus to prove our theorem we just need to show that

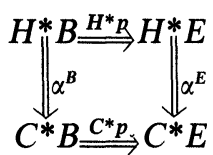

commutes in $\mathfrak{S} \mathfrak{S} \mathfrak{U}_{h}$.

This comes about by looking at the following diagram:

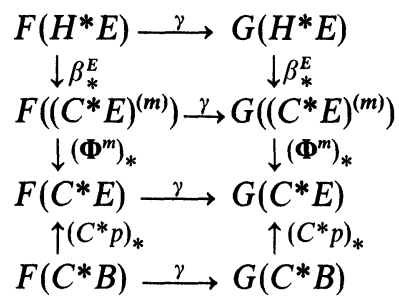

Here $F$ and $G$ are functors from shc $\mathfrak{A}$ to sets given by $F(A)=$ $\prod \mathfrak{S} \mathfrak{S} \mathfrak{U}_{h}\left(Z_{2}\left[x_{i}\right], A\right), G(A)=\mathfrak{S} \mathfrak{S P}_{h}\left(Z_{2}\left[x_{1}, \ldots, x_{n}\right], A\right)$ while $\gamma$ is the obvious natural transformation. The desired commutativity of $\left(^{*}\right)$ follows easily from the following two propositions. Let $[]=,\mathfrak{S} \mathfrak{g} \mathfrak{U}_{h}($,$) .$

Proposition 1. If $C \in \operatorname{shc} \mathfrak{A}$ and $\operatorname{deg} z=k$ then there is a bijection $c:\left[Z_{2}[z], C\right] \rightarrow H^{k}(C)$ given by

$$
c([f])=\widehat{f_{1}(z)}
$$

where a denotes the cohomology class of $a$.

Proposition 2. The diagram (**) commutes.

Proposition 1 is proved by direct computation. Proposition 2 amounts to showing that $\beta^{E}: H^{*} E \rightarrow\left(C^{*} E\right)^{(m)}$ is in shc $\mathfrak{A}$. This quickly boils down to proving that $Z_{2}\left[y_{i}\right] \rightarrow C^{*} E$ by $y_{i} \rightarrow \eta_{i}$ is in shc $\mathscr{U}$ and that in turn is an easy consequence of

Proposition 3. If $C \in \operatorname{shc} \mathfrak{A}$ then there is a bijection

given by

$$
\left[Z_{2}\left[z_{1}, z_{2}\right], C\right] \stackrel{c}{\rightarrow} H^{k_{1}} C \oplus H^{k_{2}} C \oplus H^{k_{1}+k_{2}-1} C
$$

$$
c([f])=\left(\widehat{f_{1}\left(z_{1}\right)}, \widehat{f_{1}\left(z_{2}\right)}, \widehat{\gamma(f))}\right.
$$


where $\gamma(f)=f_{2}\left(z_{1} \otimes z_{2}\right)+f_{2}\left(z_{1} \otimes z_{2}\right)+\Phi_{2}\left(\left(1 \otimes f_{1}\left(z_{1}\right)\right) \otimes\left(f_{1}\left(z_{2}\right) \otimes 1\right)\right)$, and $k_{i}=\operatorname{deg} z_{i}$.

This proposition is proved by direct computation.

Finally it is perhaps worth noting that $S q^{n-1}$ enters the discussion because $\boldsymbol{\Phi}_{2}((1 \otimes x) \otimes(y \otimes 1))+\boldsymbol{\Phi}_{2}((x \otimes 1) \otimes(1 \otimes y))$ is a perfectly good $\bigcup_{1}$-product in $C^{*} E$.

ACKNOWLEDGEMENT. The basic material concerning the categories $\mathfrak{S H} \mathfrak{A}$ and $\mathfrak{S S H}_{h}$ was developed in collaboration with V. K. A. M. Gugenheim.

\section{REFERENCES}

1. P. F. Baum, On the cohomology of homogeneous spaces, Topology 7 (1968), 15-38. MR 36 \# 2168

2. A. Borel, Sur la cohomologie des espaces fibrés principaux et des espaces homogènes de groupes de Lie compacts, Ann. of Math. (2) 57 (1953), 115-207. MR 14, 490.

3. D. Husemoller, J. C. Moore and J. Stasheff, Differential homological algebra and homogeneous spaces (preprint).

4. V. K. A. M. Gugenheim and J. P. May, On the theory and application of differential torsion products, Springer Lecture Notes in Math. (to appear).

5. J. P. May, The cohomology of principal bundles, homogeneous spaces, and two-stage Postnikov systems, Bull. Amer. Math. Soc. 74 (1968), 334-339. MR 39 \#953.

6. C. Schochet, $A$ two-stage Postnikov system where $E_{2} \neq E_{\infty}$ in the Eilenberg-Moore spectral sequence, Trans. Amer. Math. Soc. 157 (1971), 113-118.

7. J. Stasheff and S. Halperin, Differential algebra in its own rite, Proc. Adv. Study Inst. Alg. Top., Vol. III, Aarhus 1970.

Matematisk Institut, Aarhus Universitet, Aarhus, Denmark (current address) 